\title{
Fault Monitoring of the Electric Machine in a Hybrid Vehicle
}

\author{
Christofer Sundström*, Erik Frisk*, and Lars Nielsen* \\ * Vehicular Systems, Dept. of Electrical Engineering, Linköping \\ University, SE-58183 Linköping, Sweden, \{csu,frisk,lars\}@isy.liu.se.
}

\begin{abstract}
A diagnosis system for the electric machine and the power electronics in a hybrid electric vehicle is designed, where the diagnosis system uses a map based model of the system to be monitored. Such a model gives an accurate description of the fault free system, and is therefore suited for fault detectability. However, one drawback using such a model for diagnosis is that fault isolation often requires that the model, in addition to the fault free case, also describes the faulty system, and thereby measurements of the faulty system are needed, which is costly. Another approach is to use a model including physical parameters of interest in the system to be monitored, to also describe the faults' impact on the system. To achieve good diagnostic performance such a model needs to be accurate, which also is costly. Therefore, in the new approach taken here, two models for the system are used in combination to achieve good fault detectability and isolability; one is a map based model, and one is describing the faults of the system. It is shown that the approach works well and is a promising path to achieve both good fault detectability and isolability performance, without the need for neither measurements of a faulty system nor detailed physical modeling. In a simulation study evaluating the designed diagnosis system all faults are isolated and also accurately estimated.
\end{abstract}

Keywords: fault diagnosis; fault detection; fault isolation; electric machine; hybrid vehicle.

\section{INTRODUCTION}

Fault monitoring and diagnosis is used to detect and isolate faults in a system. There are several approaches to be used, and one of the more common is consistency based diagnosis (de Kleer et al., 1992) based on precompiled tests, or residual generators, as in Blanke et al. (2006). Such diagnosis systems compare the consistency between a model of the system to be monitored and the observations. Models are developed for different purposes and to different level of detail, and a common approach in the automotive industry is map based models, since these models are straightforward to design using measurements. One benefit of using such a map based model is that it accurately describes the outputs, whereas a model based on physical laws and descriptions always will show some discrepancies due to unmodeled phenomena. High model accuracy directly results in good fault detection performance, but one drawback with a map based model in a diagnosis system is the difficulty to isolate faults from each other, since internal physical phenomena are not described by the model. One way to achieve fault isolability is to collect data when the faults have occurred in the system to be monitored, which is a costly solution. Another approach to achieve good fault isolability performance is to use models that include physical parameters of interest in the diagnosis system, since then the model describes how different faults affect the system to be monitored. To achieve good diagnostic performance, such a model needs to be accurate including detailed physical modeling, which also is costly.

\subsection{Contributions and outline}

Here an approach is used that combines two models. One model is based on a map that describes the system to be monitored in the fault free case. The other model is a less accurate model for the fault free case, but this model can be used to model how the faults affect the system. The benefit of this approach is that data for a faulty system is not needed, and that the accuracy demands on the model used for fault modeling are lower than for designing a diagnosis system based on only this type of model.

The approach of combining two models is used in the design of a diagnosis system monitoring the power electronics and the electric machine used in a hybrid electric vehicle $(\mathrm{HEV})$. This system is considered since monitoring of the components in a vehicle powertrain is important in order to achieve high up-time of the vehicle. Further issues are safety and to avoid damaging components, especially the battery that is sensitive and costly. The models used in the diagnosis design of the electric machine are described in Section 2, and in Section 3 these models are combined to include fault models in the map based model. Based on this model, a diagnosis system is designed and evaluated in a simulation study in Section 5, and finally the conclusions are given in Section 6 .

\section{MODELS OF THE ELECTRIC MACHINE}

In HEVs mainly permanent magnet synchronous machines (PMSM) are used since this type of machine in general has higher efficiency compared to other machine types (Zhu and Howe, 2007; Mellor, 1999). A PMSM is an AC machine, 
but it is possible to use a DC source, e.g. a battery, and use power electronics to achieve an alternating current.

Two models of a PMSM are presented, that later are used to illustrate the approach in the design of the diagnosis system. The first model includes a map that describes the power losses in the machine and is presented in Section 2.1. To model how faults affect the machine and power electronics, which is not captured in the map based model, the second model is based on analytical expressions and is presented in Section 2.2.

\subsection{Map based model}

The map based model describe the power losses in the machine and the power electronics, and is based on measurements to find the difference between the electrical and mechanical powers. The map of the power losses, $P_{e m, l}^{\text {map }}$, is three dimensional taking the delivered torque, $T_{e m}$, motor speed, $\omega_{e m}$, and battery voltage, $U_{b}$, as inputs

$$
P_{e m, l}^{\text {map }}=f\left(T_{e m}, \omega_{e m}, U_{b}\right)
$$

and the power losses are given in Figure 1. There are limitations in the delivered torque from the machine, denoted $T_{e m, \min }$ in generator mode and $T_{e m, \max }$ in motor mode, that are functions of $\omega_{e m}$ and $U_{b}$. The limited torque, $T_{\text {em,lim }}$, is equal to the requested torque, $T_{e m, r e q}$, if the requested torque is within the limitations of what the machine is able to deliver

$$
T_{e m, l i m}=\left\{\begin{array}{l}
T_{e m, \min }, T_{e m, r e q}<T_{e m, \min } \\
T_{e m, r e q}, T_{e m, \min } \leq T_{e m, r e q}<T_{e m, \max } \\
T_{e m, \max }, T_{e m, r e q} \geq T_{e m, \max }
\end{array}\right.
$$

The delivered torque is computed by filtering $T_{\text {em }, \mathrm{lim}}$

$$
T_{e m}=\frac{1}{\tau_{e m} s+1} T_{e m, l i m}
$$

and the mechanical power delivered by the machine

$$
P_{e m, m}=T_{e m} \omega_{e m}
$$

is used to calculate the electrical power

$$
P_{e m, e}=P_{e m, m}+P_{e m, l}^{m a p}
$$

\subsection{Analytical model}

A PMSM can be modeled as a separately excited DC motor with constant field (Guzzella and Sciarretta, 2007), since the stator of a PMSM consists of windings, and the armature of permanent magnets. This is done in the model based on analytical expressions, where the resistive and frictional losses are modeled to represent the losses of the machine. The torque $T_{e m}$ is modeled to be proportional to the current, $I_{e m}$, except for the frictional losses that are modeled to be proportional to $\omega_{e m}$ (Zhu et al., 2000). The output torque from the machine is

$$
T_{e m}=k I_{e m}-c_{f} \omega_{e m}
$$

where $c_{f}$ is a friction constant and $k$ is a machine constant. The current is calculated using the voltage, $U_{e m}$, supplied by the power electronics and the electromotive force (emf), that depends on the speed of the machine, $\omega_{e m}$

$$
I_{e m}=\frac{1}{R_{e m}}(U_{e m}-\underbrace{k \omega_{e m}}_{e m f})
$$

where $R_{e m}$ is the resistance in the electric machine. The power losses in the machine are computed using

$$
P_{e m, l}^{a}=U_{e m} I_{e m}-T_{e m} \omega_{e m}
$$

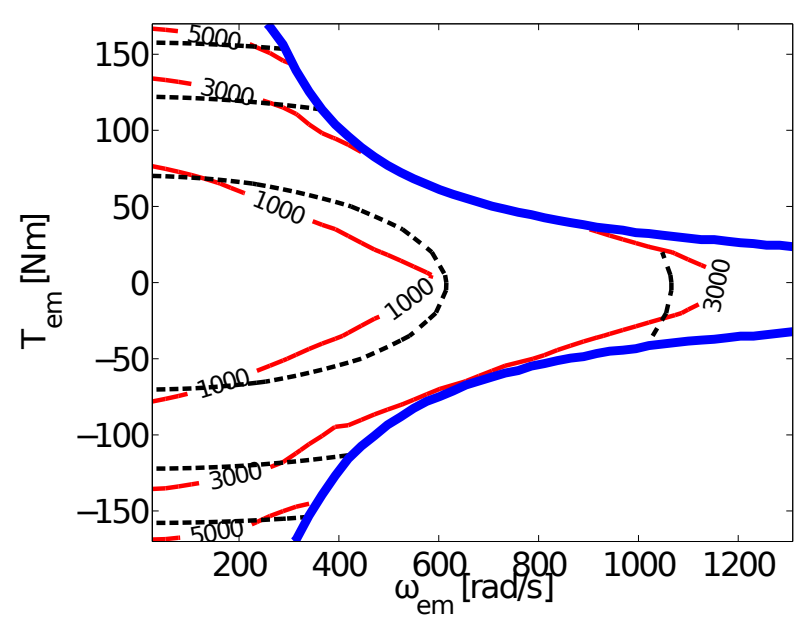

Fig. 1. The power losses [W] of the machine and power electronics. The solid (thin) red lines show the measured losses in the map described in Section 2.1, the dashed lines the losses in the model described in Section 2.2, and the solid (thick) blue line the torque limitation of the machine.

Substituting $U_{e m}$ and $I_{e m}$ from (6) and (7) gives

$$
P_{e m, l}^{a}=R_{e m}\left(\frac{T_{e m}^{2}}{k^{2}}+\frac{2 c_{f}}{k^{2}} \omega_{e m} T_{e m}+\frac{c_{f}^{2}}{k^{2}} \omega_{e m}^{2}\right)+c_{f} \omega_{e m}^{2}
$$

This model is fitted to the data of the losses given in Section 2.1. Using least squares of (9) results in that the parameters $k, R_{e m}$, and $c_{f}$ are found to be $0.50 \mathrm{Nm} / \mathrm{A}$, $0.065 \Omega$, and $0.0029 \mathrm{Nm} / \mathrm{s}$, respectively. The battery voltage is assumed to be the open circuit voltage, i.e. $250 \mathrm{~V}$, when using the map to find the losses. The power losses computed in (9) are compared with the measured losses in Figure 1.

Controller A torque from the electric machine is requested from the energy management operating on vehicle level. The controller of the machine computes a requested voltage, $U_{e m}^{c t r l}$, from the power electronics in order for the machine to, if possible, deliver this torque.

The model for the power electronics supplies this voltage to the machine when the component is fault free, i.e.

$$
U_{e m}=U_{e m, c t r l}
$$

\section{COMBINING THE MAP AND ANALYTICAL MODELS FOR FAULT MODELING}

Three fault modes are modeled in this section to be used in the design of the diagnosis system as described in Section 5, and to be used to evaluate the diagnosis system in simulations. The faults to be modeled in the electric machine affect the resistance of the machine, and the lumped torque and speed constant $k$ used in the analytical model. A fault in the power electronics results in that the applied voltage on the electric machine is not the requested voltage. The faults are modeled as

$$
\begin{aligned}
k & =k^{n o m}\left(1+f_{e m, k}\right) \\
R_{e m} & =R_{e m}^{n o m}\left(1+f_{e m, R}\right) \\
U_{e m} & =U_{e m}^{N F}\left(1+f_{p e}\right)
\end{aligned}
$$

where $k^{\text {nom }}$ and $R_{e m}^{\text {nom }}$ are the nominal values of the parameters, and $U_{e m}^{N F}$ the delivered voltage from the power 


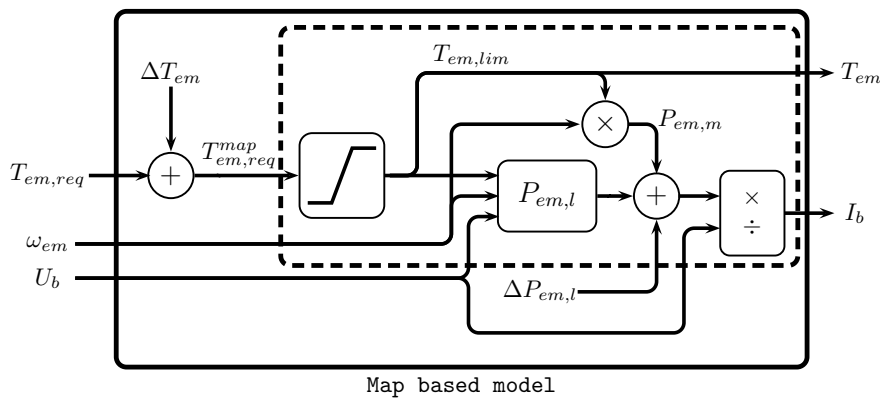

Fig. 2. The map based model includes a limitation in the torque signal, since the machine has limitations in the torque it is capable to deliver. The battery current is calculated from the mechanical power and the power losses. The modifications in the operating modes and the efficiency of the machine due to faults in the component are modeled with $\Delta T_{e m}$ and $\Delta P_{e m, l}$. The part inside the dashed line is the nominal model with no functionality for fault modeling.

electronics in the fault free case. These faults are important to monitor since they affect the delivered torque from the electric machine, as can be seen by combining (6) and (7) for the analytical model

$$
T_{e m}^{a}=k\left(\frac{U_{e m}}{R_{e m}}-\frac{k}{R_{e m}} \omega_{e m}\right)-c_{f} \omega_{e m}
$$

As stated above, the map based model is beneficial to use to represent the machine since it has high nominal accuracy, but the model has the disadvantage that the parameters affected when a fault has occurred are not included in the model. In the fault free case, the map based model of the machine delivers the requested torque, as long as the machine is capable of delivering the torque, as can be seen in the schematic structure of the model in Figure 2. The battery current, $I_{b}$, is calculated using the mechanical power, $P_{e m, m}$, and the power losses, $P_{e m, l}$, that is a map and depends on the operating points of the machine, as described in Section 2.1.

The two main ways to model faults in the map based model is to modify the input signals used in the map or to modify the output of the map. The model is here extended to modify the delivered torque from the machine when a fault has occurred, by modifying the requested torque using $\Delta T_{e m}$ according to Figure 2 . This results in that the power losses of the machine changes when there is a fault affecting the delivered torque. A fault affecting the power losses of the machine affect the current to the battery, and is modeled using $\Delta P_{e m, l}$.

In the analytical model of the electric machine described in Section 2.2, it is possible to easily induce the faults described in (11) since these parameters are included in the model. The accuracy is however generally lower in this category of models compared to the map based model. Therefore, the map based model is used to model the fault free case, and the other model is used to model the influence of the faults on the electrical machine. Expressions for $\Delta T_{e m}$ and $\Delta P_{e m, l}$ as functions of the fault modes in (11) are derived below based on the analytical model.

\subsection{Finding an expression for $\Delta T_{\text {em }}$}

From (12) it is seen that all three fault modes in (11) affect the delivered torque of the electric machine. This is described by modifying the requested torque to the fault free map based model according to

$$
T_{e m, r e q}^{\text {map }}=T_{e m, r e q}+\Delta T_{e m}
$$

where $T_{e m, r e q}$ is the requested torque from the energy management, and $\Delta T_{e m}$ is the difference between $T_{e m}$ and $T_{e m, r e q}$ due to a fault in the system. The torque delivered by the faulty machine is computed using (12), and the delivered torque in the fault free case, $T_{e m}^{a, N F}$, is also computed using (12), but with the nominal values of the parameters in the machine. The parameters $k$ and $R_{e m}$, and the voltage $U_{e m}$ used to calculate $T_{e m}^{a}$, include models for the faults according to (11), and $\Delta T_{e m}$ is expressed by

$$
\begin{aligned}
\Delta T_{e m} & =T_{e m}^{a}-T_{e m}^{a, N F} \\
& =\frac{k}{R_{e m}}\left(U_{e m}-k \omega_{e m}\right)-\frac{k^{n o m}}{R_{e m}^{n o m}}\left(U_{e m}^{N F}-k^{n o m} \omega_{e m}\right)
\end{aligned}
$$

The voltage $U_{e m}^{c t r l}$ needs to be calculated to find $U_{e m}$ and $U_{e m}^{N F}$ used in (14). This voltage is however not modeled in the map based model, and is therefore computed using the analytical model. The angular speed and the requested torque are used in the expression, that is based on (12)

$$
U_{e m}^{c t r l}=\left(\frac{T_{e m, r e q}}{k^{n o m}}+\frac{c_{f}}{k^{n o m}} \omega_{e m}\right) R_{e m}^{n o m}+k^{n o m} \omega_{e m}
$$

The delivered torque from the machine is not known in the controller of the machine, where $U_{e m}^{c t r l}$ is set, and therefore the requested torque is used in (15). For the same reason $R_{e m}$ and $k$ in the expression are the nominal values even if there is a fault in the machine affecting these parameters.

\subsection{Finding an expression for $\Delta P_{e m, l}$}

The expression for the power losses in the analytical model is given in (9), and the expression states that $f_{e m, k}$ and $f_{e m, R}$ affect the power losses in the model. The losses in the map based model are modeled as

$$
\tilde{P}_{e m, l}^{m a p}=P_{e m, l}^{m a p}+\Delta P_{e m, l}
$$

where $P_{e m, l}^{\text {map }}$ is the original map and $\Delta P_{e m, l}$ describes the difference in the power losses in the machine in the fault free and faulty cases of the machine. The losses in the faulty case are computed by (9), and the losses in the fault free case, $P_{e m, l}^{a, N F}$, are also computed by (9), but with the nominal values of the parameters $R_{e m}$ and $k$. The torque used in the computations of $P_{e m, l}^{a}$ and $P_{e m, l}^{a, N F}$ is the delivered torque $T_{e m}$ from the machine in the map based model, see Figure 2. $\Delta P_{e m, l}$ is computed by

$$
\begin{gathered}
\Delta P_{e m, l}=P_{e m, l}^{a}-P_{e m, l}^{a, N F} \\
=R_{e m}\left(\frac{T_{e m}^{2}}{k^{2}}+\frac{2 c_{f}}{k^{2}} \omega_{e m} T_{e m}+\frac{c_{f}^{2}}{k^{2}} \omega_{e m}^{2}\right)+c_{f, e m} \omega_{e m}^{2}- \\
-\left[R_{e m}^{n o m}\left(\frac{T_{e m}^{2}}{\left(k^{n o m}\right)^{2}}+\frac{2 c_{f}}{\left(k^{n o m}\right)^{2}} \omega_{e m} T_{e m}+\frac{c_{f}^{2}}{\left(k^{n o m}\right)^{2}} \omega_{e m}^{2}\right)+c_{f, e m} \omega_{e m}^{2}\right] \\
=\left(\frac{R_{e m}}{k^{2}}-\frac{R_{e m}^{n o m}}{\left(k^{n o m}\right)^{2}}\right)\left(T_{e m}^{2}+2 c_{f} \omega_{e m} T_{e m}+c_{f}^{2} \omega_{e m}^{2}\right)
\end{gathered}
$$




\section{ISOLABILITY GAIN BY COMBINING MODELS}

In this section the maximum theoretical isolability performance of a diagnosis system based on the map based model is discussed. Firstly, the isolability performance using only the map based model is considered, i.e. the results from Section 3 are assumed not to be known. Secondly, the performance when combining the map based model with the fault models obtained in Section 3 are considered.

\subsection{Theoretical fault isolability using map based model}

First, consider the case when using only the map based model, without any fault models. There are three fault modes to be monitored, see (11), and a single-fault assumption is made in the diagnosis system design. On the basis of using only the map based model, it is reasonable that all three faults affect the delivered torque

$$
T_{e m}=g_{1}\left(f_{e m, k}, f_{e m, R}, f_{p e}\right)
$$

while the power losses only depend on the fault modes in the electric machine

$$
P_{e m, l}=g_{2}\left(f_{e m, k}, f_{e m, R}\right)
$$

Note that $g_{1}$ and $g_{2}$ also depend on other variables.

When equation (19) for $P_{e m, l}$ is not consistent, this can be explained by either of the two faults $f_{e m, k}$ or $f_{e m, R}$. The fault $f_{p e}$ can not be the cause since it does not affect the power losses. If the equation for $T_{e m}$ is inconsistent, this can be explained with any of the faults according to (18). Therefore a fault in the electric machine can be isolated from a fault in the power electronics, but not vice versa. Further, it is not possible to isolate the fault modes in the electric machine from each other when no fault models are used, since both these faults affect the same model equations.

\subsection{Theoretical fault isolability using a combined model}

Now consider the case where fault models are used in combination with the map. As stated in Section 4.1, fault models are required to isolate the fault modes from each other in the diagnosis system. Here the faults' influence on $\Delta T_{e m}$ and $\Delta P_{e m, l}$ described in (14) and (17) are used in the diagnosis system. It is assumed that the faults are constant or slowly varying, and is modeled as $\dot{f}=0$. Note that the parameters $k$ and $R_{e m}$, and the voltage $U_{e m}$ all are included in the expression for $T_{e m}$, and that the faults affect the torque in different ways. This means that full fault isolability can be achieved using only information about how $T_{e m}$ is modified i.e. by only using (11) and (14). The information from how the faults affect $\Delta P_{e m, l}$ is however also used in the estimation of the faults using observers in the next section.

\section{DESIGN OF DIAGNOSIS SYSTEM}

A diagnosis system monitoring the power electronics and the electric machine of the HEV is designed based on the models presented in Sections 2 and 3. The model used, including the fault models, is first transformed into state space form in Section 5.1. This model is used to design the observer based residual generators in Section 5.2.

\subsection{State space formulation of the model}

The fault free model, described in Section 2.1, used in the design of the diagnosis system is static since the dynamics in (3) is considered fast and its dynamics is assumed instantaneous. The faults are included as states that later are estimated in the observers. In its original form the model is a DAE of differential index one (Petzold and Ascher, 1998), but is reformulated as an ODE to be able to use standard observer techniques. The model is in the form

$$
\begin{aligned}
\dot{x}_{1} & =0 \\
0 & =g\left(x_{1}, x_{2}, u\right) \\
y & =h\left(x_{1}, x_{2}, u\right)
\end{aligned}
$$

where $x_{1}$ is the vector of the dynamic variables, which is equal to the three faults, $x_{2}$ is the vector of algebraic variables, and $u$ is the vector of known input signals. The expression $g\left(x_{1}, x_{2}, u\right)$ includes the model equations, and, since the DAE has differential index one, the algebraic variables $x_{2}$ can be computed from $g\left(x_{1}, x_{2}, u\right)$ by

leading to the ODE

$$
x_{2}=g^{-1}\left(x_{1}, u\right)=G\left(x_{1}, u\right)
$$

$$
\begin{aligned}
\dot{x}_{1} & =0 \\
y & =h\left(x_{1}, G\left(x_{1}, u\right), u\right)
\end{aligned}
$$

which has the same solution set as (20). The algebraic variables $x_{2}$ and $G\left(x_{1}, u\right)$ are given by

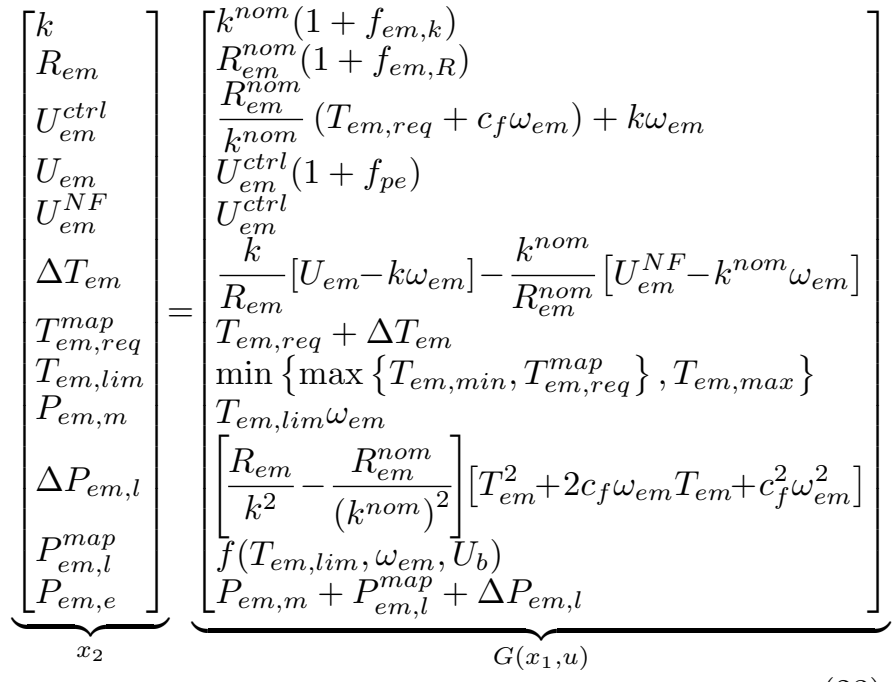

The known signals are the requested torque, angular speed, and the battery voltage. Of these the angular speed and battery voltage are sensor signals

$$
u=\left[\begin{array}{l}
T_{e m, r e q} \\
\omega_{e m} \\
U_{b}
\end{array}\right]
$$

The output signals are the delivered torque and the battery current, that are calculated in (20c) and are given by

$$
h\left(x_{1}, x_{2}, u\right)=\left[\begin{array}{c}
T_{e m, l i m} \\
\frac{P_{e m, e}}{U_{b}}
\end{array}\right]
$$

\subsection{Design of residual generators and test quantities}

The residual generators used in the diagnosis system are based on the estimated faults computed in observers using 
the model presented in Section 5.1. One way to estimate the fault modes to be monitored would be to design an observer estimating all three faults simultaneously. However, to simplify the observer design and increase the performance, the single-fault assumption is used and three observers are designed to estimate one fault each. In each observer it is assumed that the other two faults are zero, and the three observers estimating $f_{e m, k}, f_{e m, R}$, and $f_{p e}$, are denoted $\mathcal{O}_{e m, k}, \mathcal{O}_{e m, R}$, and $\mathcal{O}_{p e}$ respectively. The basic idea to achieve fault detection and isolation based on these observers is described below, but first the observers are briefly described.

Observers The observers estimating the faults used in the residual generators are designed using Extended Kalman Filters (EKF) (Kailath et al., 2000). All three observers use the same model equations, except for which fault that is to be estimated, and the used model equations are given by (23). Note that two faults in (23) are assumed to be zero in the observers, and $x_{1}$ in (22a) only includes the fault that is to be estimated in the observer. The observer used to estimate the fault $f_{e m, k}$ is e.g.

$$
\mathcal{O}_{f e m, k}:\left\{\begin{array}{l}
\dot{f}_{e m, k}=0+K(y-\hat{y}) \\
y=h\left(\left[\begin{array}{c}
f_{e m, k} \\
0 \\
0
\end{array}\right], G\left(\left[\begin{array}{c}
f_{e m, k} \\
0 \\
0
\end{array}\right], u\right), u\right)
\end{array}\right.
$$

where $x_{1}=\left[\begin{array}{lll}f_{e m, k} & 0 & 0\end{array}\right]^{T}$ in $(22 \mathrm{~b})$.

The system given in (22) is time discretized in the observers. This is trivial to do since the faults are assumed to be constant, and the only dynamics in the model is therefore $\dot{x}_{1}=0$. To be able to tune estimation convergence speed, the constant states are modeled as random walk processes

$$
x_{1, t+1}=x_{1, t}+\omega_{t}
$$

where the covariance $Q_{t}$ of the noise term $\omega$ can be used to tune observer performance. The sensors assumed available in the diagnosis system are a torque sensor and a current sensor

$$
y=\left[\begin{array}{l}
T_{\text {em,sens }} \\
I_{b, \text { sens }}
\end{array}\right]
$$

The torque sensor is used for simplicity, but if it is not available it is possible to use other sensors and an extended model, see e.g. Sundström (2011), where also the entire observer design is given.

Basic idea The faults affect the system to be monitored in different ways, see (23) for details. This, in combination with the assumption of constant faults, is used in the design of the residual generators and the basic idea is illustrated by an example. When a constant fault has occurred in the power electronics resulting in that $U_{e m} \neq U_{e m}^{c t r l}, \hat{f}_{p e}$ is constant, but the estimated faults $\hat{f}_{e m, k}$ and $\hat{f}_{e m, R}$ calculated in $\mathcal{O}_{e m, k}$ and $\mathcal{O}_{e m, R}$, are dependent on the operating point of the electric machine. This is to estimate the correct values of $\Delta T_{e m}$ and $\Delta P_{e m, l}$, and is here illustrated using the expression for $\Delta T_{e m}$ in (14). It is only $T_{e m}^{a}$ and not $T_{e m}^{a, N F}$ that is affected when there is a fault in the component. Combining (11) and (12) leads to

$$
\begin{aligned}
& T_{e m}^{a}=-c_{f} \omega_{e m}+k^{n o m}\left(1+f_{e m, k}\right) . \\
& \quad \cdot\left(\frac{U_{e m}^{N F}\left(1+f_{p e}\right)}{R_{e m}^{n o m}\left(1+f_{e m, R}\right)}-\frac{k^{n o m}\left(1+f_{e m, k}\right)}{R_{e m}^{n o m}\left(1+f_{e m, R}\right)} \omega_{e m}\right)
\end{aligned}
$$

A fault in the resistance is e.g. included in two terms in the expression, one that is proportional to $U_{e m}^{N F}$ and one that is proportional to $\omega_{e m}$. The fault in the power electronics is only included in the term that is proportional to the voltage. This leads to that when there is a constant fault in the power electronics, the value of $\hat{f}_{e m, R}$ varies with $\omega_{e m}$ to achieve the same value for $T_{e m}^{a}$ as $f_{p e}$ does. This information is used to construct residual generators in the diagnosis system. Note that it is only one of the three estimated faults in the observers that estimates a correct value of the fault.

Residual generators and decision structure Residual generators are designed based on that if an observer estimates a fault $f_{i}, \hat{f}_{i}$ converges to $f_{i}$, and that $\dot{f}_{i}=0$, but if a fault $f_{j} \neq f_{i}$ occurs it is not possible to state anything about the value $\hat{f}_{i}$ will take. Therefore, if $\dot{\hat{f}}_{i} \neq 0$, this can only be explained with that there is a fault in the system and that this fault is not $f_{i}$. This idea is used in the design of three residual generators that are based on the change in the estimated faults in the observers between two time steps. An expression for this is found in the feedback term in the observers

$$
r_{t}=K_{t}\left(y_{t}-\hat{y}_{t}\right)
$$

The residuals are post processed to form test quantities using the CUSUM algorithm (Page, 1954; Gustafsson, 2000)

$$
T\left(t_{k}\right)=\max \left\{0, T\left(t_{k-1}\right)+\left|r\left(t_{k}\right)\right|-\nu\right\}
$$

where $\nu$ is a design parameter that corresponds to the noise and model uncertainty in the residuals. The test reacts when $T$ is above a threshold $J$.

The decision structure includes information about which faults the different tests are expected to react to and is shown in Table 1. If e.g. $T_{1}$ has reacted this can be explained by $f_{e m, R}$ or $f_{p e}$, but if also $T_{2}$ has reacted the only single fault diagnosis is a fault in the power electronics. The tests $T_{1}, T_{2}$, and $T_{3}$ are based on $\mathcal{O}_{e m, k}, \mathcal{O}_{e m, R}$, and $\mathcal{O}_{p e}$ respectively.

Table 1. Decision structure for the diagnosis system including fault models. Full isolability is structurally achieved, since a unique set of tests ideally react for each fault.

\begin{tabular}{c|ccc} 
& $f_{e m, k}$ & $f_{e m, R}$ & $f_{p e}$ \\
\hline$T 1$ & & $\mathrm{X}$ & $\mathrm{X}$ \\
$T 2$ & $\mathrm{X}$ & & $\mathrm{X}$ \\
$T 3$ & $\mathrm{X}$ & $\mathrm{X}$ & \\
\hline
\end{tabular}

\subsection{Simulation results}

Simulations are carried out to exemplify that it is possible to design a diagnosis system based on the two models of the electric machine described in Section 2. The faults are induced one by one in the vehicle model described in Sundström (2011) where the map based model is used to represent the electric machine. The faults induced in the model are $f_{e m, k}=-3 \%, f_{e m, R}=-3 \%$, and $f_{p e}=-1 \%$, respectively.

The values of the thresholds $J$ are set to values for the test quantities to be well below their corresponding thresholds in the fault free case, and all monitored faults in the diagnosis 

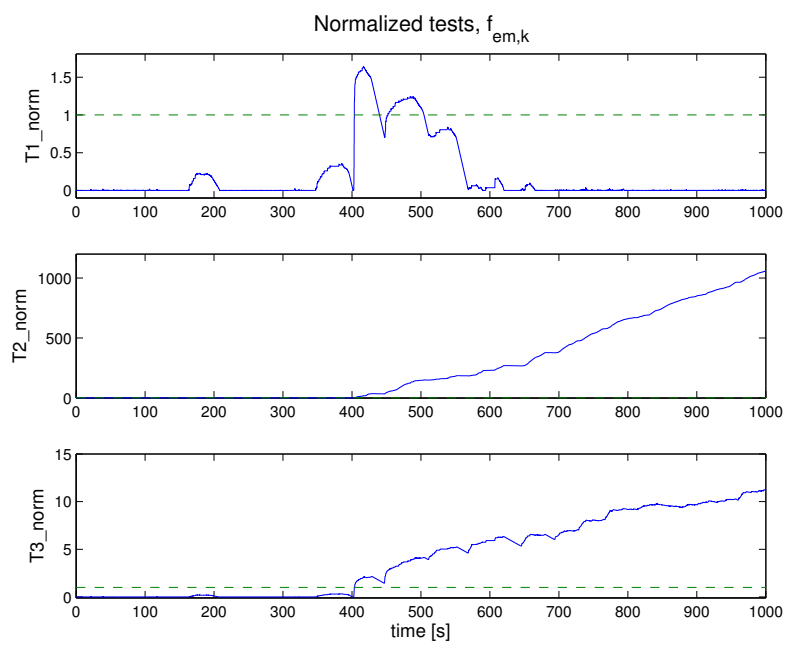

Fig. 3. The normalized test quantities when $f_{e m, k}$ has occurred after 400 seconds in FTP75. Test 2 and Test 3 are supposed to react on the fault and do so, but also Test 1 reacts for a short period immediately after the fault is induced. This can be explained with that the assumption $\dot{f}=0$ is not fulfilled when the fault occurs.

system are detected and fully isolated. Figure 3 shows the normalized test quantities

$$
T_{\text {norm }}=\frac{T}{J}
$$

when $f_{e m, k}$ is induced in the model after 400 seconds when the driving cycle FTP75 is used. According to the decision structure in Table 1, Test 2 and Test 3 are supposed to react on this fault. In Figure 3 it can be shown that also Test 1 reacts on the fault for a short time. The reason for this is that when the fault is induced, the assumption $\dot{f}=0$ is no longer valid, and the residual is non-zero since $\hat{f}_{e m, k}$ in $\mathcal{O}_{e m, k}$ has not converged to the value of the fault. This can be solved by e.g. adding a constraint that a test has to be above the threshold a predefined time before the test alarms.

The estimated faults in the three observers when $f_{e m, k}$ is induced in the vehicle model, are presented in Figure 4. It can be seen that $\hat{f}_{e m, k}$ converges fast to the correct value. It can also be seen that $\hat{f}_{p e}$ does not vary as much as $\hat{f}_{e m, R}$ when $f_{e m, k}$ is induced. This can be explained by that $U_{e m}$ and $k \omega_{e m}$ in (14) are of the same order of magnitude in most operating points. An increase in $\Delta T_{e m}$ can e.g. be achieved by increasing $U_{e m}$ or decreasing $k \omega_{e m}$. When large torques are delivered by the electric machine, the difference between $U_{e m}$ and $k \omega_{e m}$ is larger, and the term $\frac{k}{R_{e m}}$ in (14) has larger influence on $\Delta T_{e m}$. Therefore it is possible to isolate the faults $f_{p e}$ and $f_{e m, k}$ from each other.

\section{CONCLUSIONS}

A method combining two models, one with good nominal accuracy and one with the possibility to model how the faults affect the system, was used in the design of a diagnosis system for the power electronics and the electric machine in an HEV. It was shown that the method works well and is a promising path to achieve both good fault detectability and isolability performance, but with no need of measurements
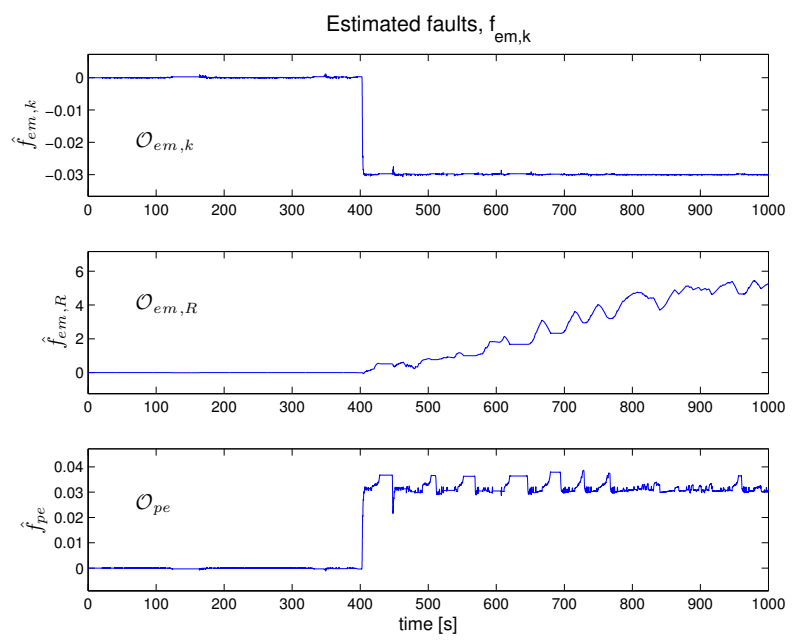

Fig. 4. The estimated faults in the three observers when $f_{\text {em }, k}=-3 \%$ after 400 seconds in FTP75. The estimated fault $\hat{f}_{e m, k}$ converges fast to the value of $f_{e m, k}$, but $\hat{f}_{e m, R}$ and $\hat{f}_{p e}$ varies significantly since these estimated faults are computed in observers not considering the fault that has occurred according to Section 5.2.

of a faulty system or detailed physical modeling of the faulty cases. In addition to fault isolation, the observers used in the diagnosis system accurately estimates the size of the faults.

\section{REFERENCES}

Blanke, M., Kinnaert, M., Lunze, J., and Staroswiecki, M. (2006). Diagnosis and Fault-Tolerant Control. Springer, 2nd edition.

de Kleer, J., Mackworth, A., and Reiter, R. (1992). Characterizing diagnoses and systems. Artificial Intelligence, $56(2-3), 197-222$.

Gustafsson, F. (2000). Adaptive filtering and change detection. John Wiley \& Sons.

Guzzella, L. and Sciarretta, A. (2007). Vehicle Populsion System, Introduction to Modelling and Optimization. Springer Verlag, Zürich, 2nd edition.

Kailath, T., Sayed, A.H., and Hassibi, B. (2000). Linear Estimation. Prentice Hall.

Mellor, P. (1999). High efficiency drive-trains for electric and hybrid vehicles. In Electrical Machine Design for All-Electric and Hybrid-Electric Vehicles (Ref. No. 1999/196), IEE Colloquium on, 8/1 -8/7.

Page, E. (1954). Continuous inspection schemes. Biometrika, 41, 100-115.

Petzold, L.R. and Ascher, U.M. (1998). Computer Methods for Ordinary Differential Equations and DifferentialAlgebraic Equations. Siam.

Sundström, C. (2011). Vehicle level diagnosis for hybrid powertrains. Technical report. LiU-TEK-LIC-2011:27, Thesis No. 1488.

Zhu, G., Dessaint, L.A., Akhrif, O., and Kaddouri, A. (2000). Speed tracking control of a permanent-magnet synchronous motor with state and load torque observer. Industrial Electronics, IEEE Trans. on, 47(2), 346 -355.

Zhu, Z. and Howe, D. (2007). Electrical machines and drives for electric, hybrid, and fuel cell vehicles. Proceedings of the IEEE, 95(4), $746-765$. 